\title{
Health factors and spinal cord injury: a prospective study of risk of cause-specific mortality
}

\author{
Yue $\mathrm{Cao}^{1} \cdot$ Nicole DiPiro $^{1} \cdot$ James S. Krause ${ }^{1}$
}

Received: 30 November 2018 / Revised: 8 February 2019 / Accepted: 8 February 2019 / Published online: 25 February 2019

(c) International Spinal Cord Society 2019

\begin{abstract}
Study design Prospective cohort study.

Objectives: Identify the risk and protective factors of all-cause and cause-specific mortality among persons with traumatic spinal cord injury (SCI).

Setting Rehabilitation specialty hospital in the Southeastern United States.

Methods A prospective cohort study was conducted with 3070 adults with traumatic SCI who were a minimum of 1 year post-injury at assessment. Prospective data were collected in 1997-1998 and 2007-2010, with mortality determined as of 31 December, 2016. The deceased were classified into six categories based on underlying cause of death: septicemia, pneumonia and influenza, cancer, heart and blood vessel diseases, unintentional injuries, and all other causes. The competing risk analysis strategy applied to each of the specific causes.

Results There were a total of 803 observed deaths among the 2979 final study sample. After controlling for demographic and injury characteristics, general health, pressure ulcer history, and symptoms of infections were significantly associated with all-cause mortality. Except for cancer, they were also related with at least one of the specific causes of death, whereas orthopedic complications and subsequent injuries were unrelated to any cause.

Conclusions Three health domains, global health, pressure ulcers, and symptoms of illness or infection, were significantly associated with mortality after SCI, and the patterns of association varied as a function of specific cause of death.
\end{abstract}

\section{Introduction}

Individuals with spinal cord injury (SCI) have an increased risk of mortality compared to the general population [1-4]. Despite advancements in acute survival [1, 2, 5], mortality rates for those with chronic injury ( $>1$ year) have not improved since the 1980s [3,6]. Several specific causes and risk factors contribute to the excess mortality and reduced life expectancy observed in individuals with chronic SCI. The leading causes of death, based on data from the 2017 SCI Model Systems Annual Report, include diseases of the respiratory system (22\%; $65 \%$ of which are pneumonia), infectious and parasitic disease $(12 \% ; 90 \%$ of which are cases of septicemia), cancer $(10.2 \%)$, hypertensive and

Yue Cao

caoyu@musc.edu

1 College of Health Professions, Medical University of South Carolina, Charleston, SC 29403, USA ischemic heart disease (10\%), other heart disease $(8.4 \%)$, and unintentional injuries $(6.6 \%)$ [3]. These prevalence rates differ somewhat from recent reports from other countries; however, the leading causes of death remain relatively consistent [7-9]. In the United States, while it appears that mortality rates are declining for some causes (e.g., diseases of the cardiovascular system, cancer, and stroke), increases in the prevalence rates of other causes (e.g., unintentional injuries) are offsetting overall gains in life expectancy [10]. No change in deaths due to respiratory issues or septicemia have been reported. These trends in mortality highlight the need for continued examination of the factors that contribute to cause-specific mortality in those with chronic SCI.

Much of the research on mortality after SCI has been limited to all-cause mortality and the demographic and injury characteristics influencing survival. Elevated risk of mortality is associated with injury severity, specifically higher neurologic level and completeness of SCI, older age at injury, and etiology of injury [4, 11, 12]. Significant disparities in mortality and survival based on SCI 
characteristics were recently reported. These disparities increased with longer time since injury [11]. The authors concluded there is a need to further investigate causespecific mortality and modifiable health factors that contribute to the observed disparities.

Modifiable risk factors have been previously studied. Based on the Theoretical Risk and Prevention Model (TRPM) developed by Krause et al. [13, 14], associations between mortality and diverse classes of risk factors have been identified in individuals with chronic SCI. Extending beyond basic demographic and injury factors directly related to mortality, the multi-stage TRPM provides a framework for successive sets of potentially modifiable risk and protective factors for mortality, including psychological and socio-environmental factors, behavioral factors, and health and secondary conditions. Health factors are the immediate risk factors of mortality [14]. These sets of factors have been the focus of several studies of mortality after SCI [15-26], but have only recently begun to be examined in relation to cause-specific mortality [15].

Considering the shift in the leading causes of death among those with SCI and the continued high rates of respiratory and septicemia-related deaths, it is important to understand how specific factors, beyond demographic and SCI characteristics, may influence cause-specific death. An earlier multi-stage analysis, using a prospective cohort design, assessed the relationship between health factors and elevated risk of all-cause mortality [19]. Several health outcomes were associated with mortality. General health indices, treatments, subsequent injuries, probable major depression, pressure ulcers, and several outcomes within the biologic domains of symptoms of SCI complications and irreversible conditions were significantly associated with elevated hazard of mortality. Only selected health factors that predicted the greatest amount of unique variance were included in the final model. Days spent in the hospital, number of infection symptoms, fracture or amputation since injury, pressure ulcer repair surgeries, and probable major depression were all significantly associated with a higher hazard of mortality in the final model [19]. Subsequent studies have supported these health factors, as well as history of chronic pressure ulcers, as predictors of mortality among those with chronic SCI [15, 22, 27].

Studies linking multiple risk factors, beyond demographic and injury characteristics, with cause-specific mortality have been rare. A recent analysis of existing data from the SCI Model Systems identified several healthrelated factors of cause-specific mortality [15]. Unfortunately, the findings are limited by retrospective analysis of existing data. The authors indicate the need for cohort studies "with a wider range of predictive factors, particularly health and environmental factors that may directly affect hazard of mortality" [15, p. 1677]. Another study identified risk factors for a highly specific cause of death, unintentional deaths due to drug poisoning (i.e., overdose), finding several behaviors and a personality trait reflective of impulsivity related to an elevated risk of mortality [28]. Clearly, prospective studies are needed to identify predictors of cause-specific mortality.

\section{Purpose}

Our purpose was to identify risk and protective health factors associated with all-cause and cause-specific mortality after SCI. This study builds upon previous research by using five health factors identified in a structural equation modeling (SEM) analysis of health outcomes as predictors, after controlling for other demographic and injury characteristics [29]. The objectives were to identify the relationship of the five health domains (global health, pressure ulcers, symptoms of illness or infection, orthopedic, and subsequent injuries) with: (1) all-cause mortality and (2) specific causes of death, including septicemia, pneumonia and influenza, cancer, heart and blood vessel diseases, unintentional injuries, and other causes.

\section{Methods}

\section{Participants}

Institutional review board approval was obtained prior to initiating the study. Participants were identified from a specialty hospital in the Southeastern United States and enrolled in a prospective cohort study. The inclusion criteria were minimum of 18 years old at the time of survey, traumatic SCI of at least 1 year duration, and having residual effects resulting from traumatic SCI. They were enrolled on one of two occasions. The first cohort was enrolled in 1997-1998 $(n=1386)$ and the second in 2007-2009 $(n=1684)$, with a total of 3070 participants. After removing 46 ineligible participants who were fully recovered and 45 participants missing key time information, the final study sample was 2979.

\section{Prospective data collection procedures}

Letters were sent to potential participants to describe the prospective study and alert them that the self-report assessment (SRA) was forthcoming. The SRA forms were sent 4-5 weeks later, with two subsequent mailings along with follow-up phone calls for non-respondents. If individuals expressed an interest but had misplaced or discarded materials, another set of materials was mailed. In 1997-1998, participants were offered \$20 remuneration and were made eligible for drawings totaling 
$\$ 1500$, whereas they were offered $\$ 50$ remuneration in 20072009. Of the original cohort of 1386, 863 completed follow-up measures.

The National Death Index (NDI) of the National Center for Health Statistics [30] was used for determining mortality and causes of death. Mortality status was determined as of 31 December, 2016. Based on the International Classification of Diseases (ICD) codes of underlying cause of death provided by the NDI, we classified the deceased into six categories: septicemia (A40-A41), pneumonia and influenza (J09-J18), cancer (C00-D49), heart and blood vessel diseases (I00-I99), unintentional injuries (V01-X59, Y40-Y84, Y88), and all other causes (ICD-10-CM codes). There were 71 cases ( $8.8 \%$ of total deaths) for which the underlying cause of death ICD code was listed as either "Paraplegia and quadriplegia (G82)" or "Other and unspecified diseases of spinal cord (G95)." In these cases, their cause of death was reclassified based on clinical judgment using the reported contributing causes, up to 20 multiple causes. Two independent experts classified causes of death in these cases, and a third expert made the final selection in cases where there was disagreement among the first two.

\section{Measures}

The primary variables of interests included five health factors that were identified from the structural analysis of 25 health outcomes [29]. In that study, exploratory factor analysis (EFA) was applied to the first-half of the sample, and then confirmatory factor analysis (CFA) was applied to the second-half of the sample to cross-validate the EFA factor structures by specifying the number of factors and the indicators for each factor a priori. The EFA generated an excellent fit (root mean square error of approximation $=$ 0.042), while the CFA had an acceptable fit (root mean square error of approximation $=0.065$ ).

The first factor, global health, included general health ratings, days affected by poor health and poor mental health, and depressive symptoms; the pressure ulcers factor was comprised of total number of sores, current sore, reduced days of sitting due to sore, and surgeries to repair a sore; the symptoms of illness or infection factor reflected seven symptoms: stomach problems, bowel accidents, rectal bleeding, urine leaking, fevers, urinary tract infections, and sweats/chills; the orthopedic conditions factor included curvature of the spine, amputations, broken bones, and contracture; the subsequent injuries factor measured injuries requiring medical attention, total number of injuries since SCI onset, and hospitalizations for injury. A sixth factor, treatments, was not used because it reflected types of treatments (e.g., physician visits, hospitalizations), rather than conditions or global rankings. As we did not measure total number of injuries since SCI onset, the subsequent injuries factor was comprised of injuries requiring medical attention and the number of hospitalizations for injury.
We standardized each indicator and utilized the sum of $Z$-scores as the composite score for all health factors, as the health domains include multiple items in different metrics (i.e., it is not possible to provide a summative score without a transformation). Composite scores combine multiple health outcome measures, so these health factors are more informative and stable than individual indicators. Furthermore, they provide consistent and repeatable measures that were both based on the existing literature and over multiple measurements within the prospective cohort study.

We also measured gender (male vs. female), race/ethnicity (non-Hispanic White, non-Hispanic Black, and others), age at injury, years post-injury, and injury severity $(\mathrm{C} 1-\mathrm{C} 4$ level, nonambulatory; C5-C8 level, non-ambulatory; non-cervical, nonambulatory; and all ambulatory regardless of injury level).

\section{Analysis}

We first compared the demographic, injury characteristics, and five health factors between survivors and the deceased. The survival analyses were completed using Cox proportional hazards regression models with the PHREG procedure in SAS software version 9.4 [31]. The first Cox model was developed for the multivariate analyses of all-cause mortality. Besides the five health factors, we added five demographic and injury characteristics as the covariates in the Cox model. If a participant was measured in both baseline and the follow-up, we utilized their most recent response. However, if a measure at the follow-up was missing, we used their baseline measure as the replacement. All the deceased participants were censored at the time when the death occurred, and all the other participants were censored on 31 December, 2016 in our analyses.

We then applied competing risk analysis strategy by using Cox models to estimate hazard ratios (HRs) separately for each of the six specific causes of death. In each Cox model, the other causes were treated as censored at the time when the other cause occurred (one cause of death removes the individual from all the other causes) [32]. The proportional hazards assumption of each model was evaluated using the Schoenfeld residuals and found to be tenable. We also developed the cumulative incidence function (CIF) for every specific cause of death by using the SAS CIF macro. The CIF estimated the crude incidence of each specific death cause while taking competing risks into account [33].

\section{Results}

\section{Descriptive and bivariate analysis}

Among the 2979 participants, 803 were deceased (27\%), with an average 5.11 years of follow-up. By the end of 2016, there were 2176 survivors with an average 8.90 
Table 1 Descriptive and bivariate analysis

\begin{tabular}{|c|c|c|c|}
\hline & Survivors & Deceased & $p$-value \\
\hline & \multicolumn{2}{|c|}{ Column (\%) } & \\
\hline Injury severity & & & $<0.01$ \\
\hline $\begin{array}{l}\mathrm{C} 1-\mathrm{C} 4 \text {, non- } \\
\text { ambulatory }\end{array}$ & 8 & 19 & \\
\hline $\begin{array}{l}\text { C5-C8, non- } \\
\text { ambulatory }\end{array}$ & 24 & 31 & \\
\hline $\begin{array}{l}\text { Non-cervical, non- } \\
\text { ambulatory }\end{array}$ & 35 & 35 & \\
\hline All ambulatory & 32 & 16 & \\
\hline Race/ethnicity & & & 0.99 \\
\hline Non-Hispanic White & 71 & 71 & \\
\hline Non-Hispanic Black & 21 & 20 & \\
\hline Others & 9 & 9 & \\
\hline Gender & & & 0.01 \\
\hline Female & 25 & 21 & \\
\hline \multirow[t]{2}{*}{ Male } & 75 & 79 & \\
\hline & \multicolumn{2}{|c|}{ Mean \pm SD } & \\
\hline Age at injury & $31.53 \pm 13.19$ & $38.99 \pm 16.30$ & $<0.01$ \\
\hline Years post-injury & $11.61 \pm 9.24$ & $13.41 \pm 9.92$ & $<0.01$ \\
\hline \multicolumn{4}{|l|}{ Health factors } \\
\hline Global health & $0.18 \pm 1.89$ & $-0.48 \pm 2.09$ & $<0.01$ \\
\hline Pressure sores & $-0.44 \pm 2.63$ & $1.20 \pm 3.64$ & $<0.01$ \\
\hline $\begin{array}{l}\text { Symptoms of illness or } \\
\text { infections }\end{array}$ & $-0.22 \pm 3.95$ & $0.61 \pm 4.47$ & $<0.01$ \\
\hline Orthopedic conditions & $-0.04 \pm 2.84$ & $0.10 \pm 3.63$ & 0.26 \\
\hline Subsequent injuries & $-0.04 \pm 1.52$ & $0.11 \pm 1.82$ & 0.03 \\
\hline
\end{tabular}

follow-up years. The average age was 52 for those surviving at the study endpoint, and, for those deceased, the average age at death was 58. The bivariate analyses (Table 1) indicates the deceased participants were older at injury, had greater years post-injury, and were more liked to be male and more severely injured than those who survived. Compared to the deceased, the survivors had significantly higher global health scores and lower scores for pressure ulcers, illness/infections symptoms, and subsequent injuries. We did not find a significant difference in the orthopedic condition score between the two groups.

\section{Survival analysis}

In the multivariate analyses for all-cause mortality (Table 2), multiple factors were significantly associated with mortality, including three of the five health factors. Compared to ambulatory participants, those who were non-ambulatory had significantly higher risk of all-cause mortality. Male gender, age at injury onset, and years postinjury were also significant risk factors. Race/ethnicity was not significantly related to mortality.
Table 2 Multivariate analysis for all-cause mortality

$\operatorname{HR}(95 \% \mathrm{CI})$

\begin{tabular}{ll} 
Injury severity (ref = ambulatory) & \\
C1-C4, non-ambulatory & $3.39(2.67-4.31)$ \\
C5-C8, non-ambulatory & $2.10(1.69-2.62)$ \\
Non-cervical, non-ambulatory & $1.52(1.22-1.89)$ \\
Race/ethnicity (ref = others) & \\
Non-Hispanic White & $1.05(0.82-1.35)$ \\
Non-Hispanic Black & $0.89(0.67-1.18)$ \\
Gender (ref = female) & \\
Male & $1.20(1.01-1.43)$ \\
Age at injury & $1.05(1.04-1.05)$ \\
Years post-injury & $1.04(1.03-1.05)$ \\
Health factors & \\
Global health & $0.96(0.92-1.00)$ \\
Pressure sores & $1.10(1.07-1.12)$ \\
Symptoms of illness or infections & $1.02(1.01-1.04)$ \\
Orthopedic conditions & $1.01(0.99-1.03)$ \\
Subsequent injuries & $0.99(0.95-1.04)$ \\
\hline$H R$ hazard ratio, $C I$ confidence interval &
\end{tabular}

After controlling for all demographic and injury characteristics, we found higher global health scores were protective of all-cause mortality, whereas higher scores for pressure ulcers and illness/infections reflected a higher risk of all-cause mortality. The relationships between mortality and orthopedic conditions or subsequent injuries were not statistically significant.

Table 3 summarizes the association between health factors and mortality, which varied by specific cause of death. Pressure ulcers were a significant risk factor for deaths due to septicemia, heart and blood vessel diseases, unintentional injuries, and other deaths. The symptoms of illness or infections were a significant risk factor for death due to pneumonia/influenza and other deaths. The global health factor decreased the risk of deaths due to heart and blood vessel diseases and unintentional injuries. None of the health factors were significantly associated with death due to cancer.

The patterns of association with demographic and injury characteristics also varied as a function of specific cause of death. Injury severity was a significant risk factor for each cause of death except for cancer. The strengths of the relationships varied dramatically and were highest for pneumonia and influenza $(\mathrm{HR}=6.27$ for $\mathrm{C} 1-\mathrm{C} 4$, non-ambulatory) and septicemia ( $\mathrm{HR}=4.14$ for $\mathrm{C} 1-\mathrm{C} 4$ non-ambulatory). Age at injury onset and years post-injury were significant risk factors for each cause of death, except for unintentional injuries. Males were more likely to have died from heart and blood vessel diseases and all other causes. 
Table 3 Multivariate analysis for cause-specific mortality

\begin{tabular}{|c|c|c|c|}
\hline & Septicemia $(n=56)$ & $\begin{array}{l}\text { Pneumonia and influenza } \\
(n=45)\end{array}$ & Cancer $(n=83)$ \\
\hline & HR $(95 \%$ CI $)$ & HR $(95 \%$ CI $)$ & HR $(95 \% \mathrm{CI})$ \\
\hline \multicolumn{4}{|c|}{ Injury severity (ref = ambulatory) } \\
\hline C1-C4, non-ambulatory & $4.14(1.70-10.10)$ & $6.27(2.39-16.47)$ & $1.18(0.54-2.57)$ \\
\hline C5-C8, non-ambulatory & $1.94(0.82-4.58)$ & $2.77(1.08-7.12)$ & $1.00(0.54-1.83)$ \\
\hline $\begin{array}{l}\text { Non-cervical, non- } \\
\text { ambulatory }\end{array}$ & $1.34(0.58-3.12)$ & $1.34(0.50-3.58)$ & $0.83(0.46-1.49)$ \\
\hline \multicolumn{4}{|l|}{ Race/ethnicity (ref = others) } \\
\hline Non-Hispanic White & $0.95(0.37-2.43)$ & $1.99(0.49-8.08)$ & $1.05(0.48-2.32)$ \\
\hline Non-Hispanic Black & $1.30(0.47-3.57)$ & $2.75(0.63-12.06)$ & $1.18(0.49-2.82)$ \\
\hline \multicolumn{4}{|l|}{ Gender $($ ref $=$ female $)$} \\
\hline Male & $0.72(0.40-1.28)$ & $0.58(0.31-1.09)$ & $1.22(0.72-2.07)$ \\
\hline Age at injury & $1.05(1.03-1.07)$ & $1.07(1.04-1.09)$ & $1.06(1.05-1.08)$ \\
\hline Years post-injury & $1.06(1.03-1.09)$ & $1.05(1.02-1.09)$ & $1.07(1.05-1.10)$ \\
\hline \multicolumn{4}{|l|}{ Health factors } \\
\hline Global health & $1.00(0.86-1.16)$ & $1.02(0.87-1.21)$ & $0.94(0.84-1.05)$ \\
\hline Pressure sores & $1.14(1.06-1.22)$ & $1.02(0.93-1.13)$ & $1.04(0.97-1.12)$ \\
\hline $\begin{array}{l}\text { Symptoms of illness/ } \\
\text { infections }\end{array}$ & $0.99(0.92-1.06)$ & $1.11(1.03-1.19)$ & $1.00(0.94-1.06)$ \\
\hline Orthopedic conditions & $1.04(0.79-1.37)$ & $1.22(0.90-1.67)$ & $1.04(0.82-1.32)$ \\
\hline \multirow[t]{3}{*}{ Subsequent injuries } & $1.03(0.89-1.20)$ & $0.93(0.75-1.15)$ & $0.96(0.82-1.12)$ \\
\hline & $\begin{array}{l}\text { Heart and blood } \\
\text { vessel diseases }(n=172)\end{array}$ & $\begin{array}{l}\text { Unintentional } \\
\text { injuries }(n=81)\end{array}$ & $\begin{array}{l}\text { Other causes } \\
(n=366)\end{array}$ \\
\hline & $\mathrm{HR}(95 \% \mathrm{CI})$ & $\mathrm{HR}(95 \% \mathrm{CI})$ & $\mathrm{HR}(95 \% \mathrm{CI})$ \\
\hline \multicolumn{4}{|c|}{ Injury severity (ref $=$ ambulatory) } \\
\hline $\mathrm{C} 1-\mathrm{C} 4$, non-ambulatory & $2.59(1.47-4.56)$ & $2.27(1.07-4.84)$ & $5.00(3.46-7.23)$ \\
\hline C5-C8, non-ambulatory & $2.37(1.47-3.80)$ & $1.60(0.81-3.14)$ & $2.74(1.92-3.89)$ \\
\hline $\begin{array}{l}\text { Non-cervical, non- } \\
\text { ambulatory }\end{array}$ & $1.80(1.13-2.88)$ & $1.36(0.71-2.59)$ & $1.88(1.33-2.67)$ \\
\hline \multicolumn{4}{|l|}{ Race/ethnicity (ref = others) } \\
\hline Non-Hispanic White & $1.12(0.64-1.97)$ & $1.34(0.61-2.95)$ & $0.98(0.69-1.38)$ \\
\hline Non-Hispanic Black & $1.17(0.64-2.16)$ & $0.54(0.20-1.45)$ & $0.65(0.44-0.98)$ \\
\hline \multicolumn{4}{|l|}{ Gender $(\mathrm{ref}=$ female $)$} \\
\hline Male & $1.65(1.09-2.51)$ & $1.03(0.61-1.73)$ & $1.32(1.01-1.72)$ \\
\hline Age at injury & $1.06(1.05-1.07)$ & $1.01(0.99-1.03)$ & $1.05(1.04-1.05)$ \\
\hline Years post-injury & $1.06(1.04-1.07)$ & $0.98(0.95-1.01)$ & $1.03(1.02-1.04)$ \\
\hline \multicolumn{4}{|l|}{ Health factors } \\
\hline Global health & $0.91(0.84-0.98)$ & $0.90(0.80-1.01)$ & $0.99(0.94-1.05)$ \\
\hline Pressure sores & $1.08(1.04-1.13)$ & $1.09(1.02-1.16)$ & $1.12(1.09-1.15)$ \\
\hline $\begin{array}{l}\text { Symptoms of illness/ } \\
\text { infections }\end{array}$ & $1.01(0.97-1.05)$ & $1.01(0.96-1.07)$ & $1.03(1.01-1.06)$ \\
\hline Orthopedic conditions & $0.88(0.74-1.06)$ & $1.16(0.91-1.47)$ & $0.99(0.95-1.02)$ \\
\hline Subsequent injuries & $0.96(0.87-1.07)$ & $1.04(0.92-1.18)$ & $1.02(0.95-1.08)$ \\
\hline
\end{tabular}

$H R$ hazard ratio, $C I$ confidence interval

After accounting for the competing risks, we found the crude incidence for septicemia deaths was $4 \%$ at the endpoint of study, $2 \%$ for pneumonia and influenza deaths, $5 \%$ for cancer deaths, $12 \%$ for heart and blood vessel disease deaths, $5 \%$ for unintentional injury deaths, and $23 \%$ for all the other deaths (Fig. 1). 


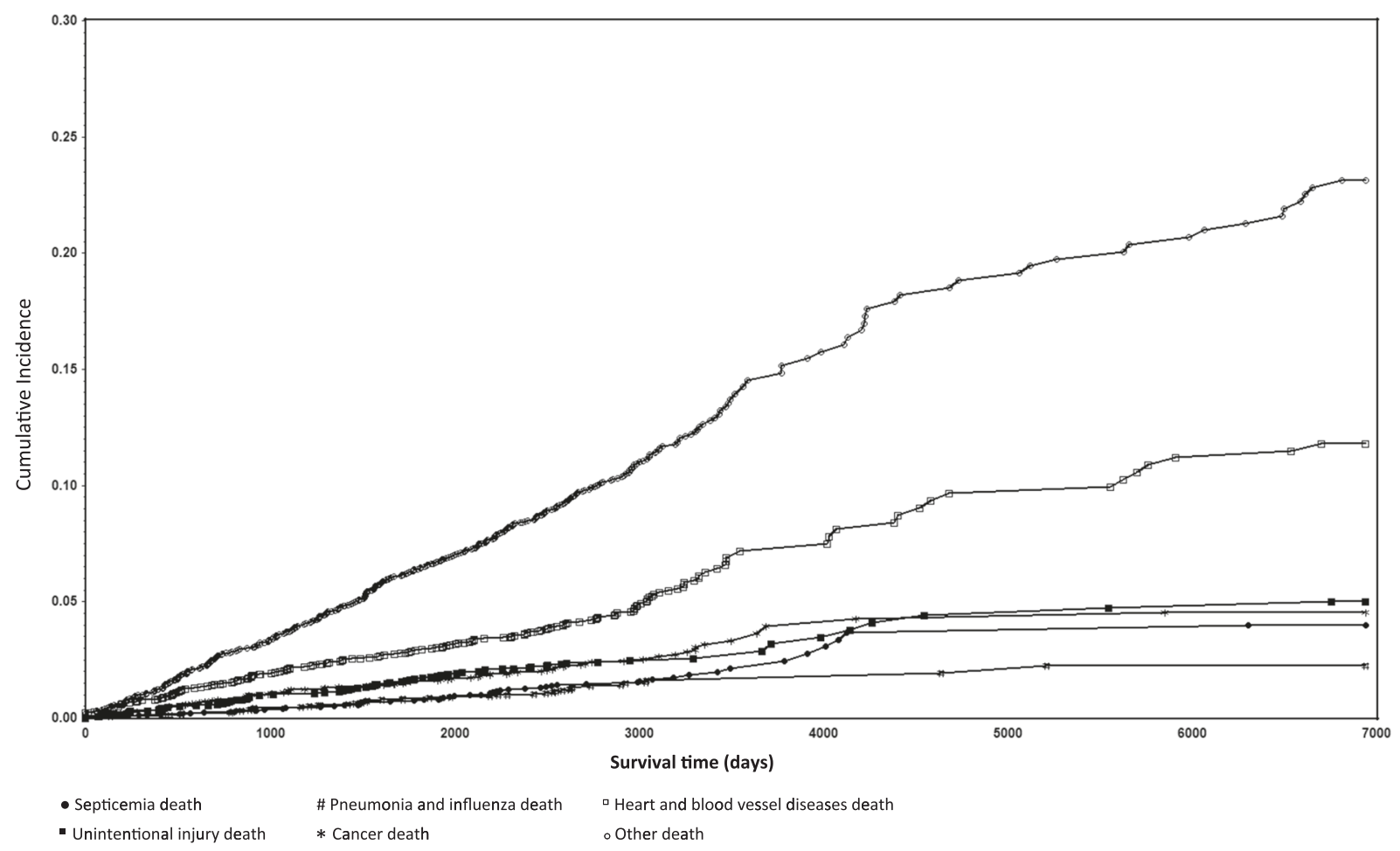

Fig. 1 Cumulative incidence function for six cause-specific death

\section{Discussion}

The unique contribution of this study was the investigation of health domains in relation to cause-specific mortality after SCI. With the exception of one recent study [15], existing studies have either measured a small number of demographic and injury factors in relation to cause-specific mortality or more diverse factors in relation to all-cause mortality. The SCI Model Systems study [15] found several health factors predictive of at least one cause of death, including self-reported health, hospitalizations, grade 3 or 4 pressure ulcer, pneumonia, and deep vein thrombosis. At least one of the five health dimensions was predictive of each cause of death, except for cancer, which was essentially unrelated to SCI (either SCI indices or secondary health complications). General health, pressure ulcer history, and symptoms of infections were significantly associated with at least one of the causes of death, whereas orthopedic complications and subsequent injuries were unrelated to any cause.

The pressure ulcer dimension was strongly associated with both all-cause and multiple individual causes of mortality (all except pneumonia/influenza and cancer). Previous research [15] has identified a significant relationship between grade 3 and grade 4 pressure ulcers and all-cause mortality, as well as mortality due to infective diseases, respiratory system diseases, heart and blood vessel diseases, external causes, and all other causes. The mechanism by which pressure ulcers may contribute to septicemia is intuitive, whereas the mechanism by which they relate to death due to unintentional injury or other causes is less clear from the current analysis. However, previous research has suggested some behavioral risk factors, such as binge drinking, regular smoking, and medication usage, that contribute to pressure ulcers also contribute to deaths related to unintentional injuries and other causes [28, 34]. The mechanism by which pressure ulcers are associated with cardiovascular disease mortality may be a more complex relationship. Among individuals hospitalized with pressure ulcers, congestive heart failure and myocardial infarction are associated with increased hazard of mortality [35]. An increased risk for pressure ulcers has also been reported in the general population among those with cardiovascular disease [36] and congestive heart failure [37] and individuals with SCI with metabolic syndrome [38]. These findings clearly suggest the importance of pressure ulcer prevention and management in reducing multiple types of mortality.

Global health also appears to be an important predictor of mortality, particularly cardiovascular disease and unintentional injury. This measure itself is comprised of general indicators of health, including mental health (i.e., depressive symptoms), so it is possible that deaths due to cardiovascular disease are related for overall health rating, whereas those related to unintentional injury may be more closely related to emotional health. Deaths due to unintentional 
injury include those related to unintentional drug poisoning, which may have a psychological component.

The finding that symptoms of infection are predictive of pneumonia and influenza only suggests that those with a history of symptoms of infection may have greater susceptibility, specifically to pneumonia and influenza. The absence of relationships between orthopedic complications, including amputations and fractures, and history of subsequent injuries in relation to mortality is surprising, given that they were significant in previous research [19]. However, their effects may be somewhat masked by being part of a larger factor.

From a clinical perspective, relationships between the health domains and cause-specific mortality highlight the potential for those from multiple disciplines and specialties to contribute to the development of intervention strategies. Reducing the risk of death due to unintentional injury will require different expertize than reducing risk of death due to infectious disease. Medical doctors, nurses, and rehabilitation professionals play an important role in evaluating an individual's history, including pressure ulcers and symptoms of illness and infection, and utilizing this information to advise the individual on proper treatment and management to attenuate the risk of mortality. For instance, the findings suggest the importance of pressure ulcer history, not only with risk of all-cause mortality, but risk of causes that are not intuitive (i.e., those due to heart and blood vessel diseases, unintentional injuries, and "other" causes).

As this study used health dimensions, rather than specific diagnoses (e.g., stage 3 or 4 pressure ulcer) the findings have more generalizability to the full pressure ulcer domain. This contrasts with recent research on cause-specific mortality where diagnoses were linked to risk of mortality using data from the SCI Model Systems in the United States [15]. Similarly, findings suggest that the domain of symptoms of infection may be important in predicting mortality. Therefore, the presence of complications such as bowel accidents, rectal bleeding, urine leaking, fevers, and sweats/chills may also be indicators of a need for developing treatment, management, or surveillance protocols to decrease the risk of all-cause mortality and death due to pneumonia and influenza and all other causes.

\section{Study strengths and limitations}

There were several important methodologic considerations, both strengths and weaknesses, including: (1) prospective cohort methodology based on the TRPM, (2) utilization of a set of health predictors that were defined in previous research, and (3) identification of competing risks of different causes of mortality. First, the prospective design and utilization of the conceptual model were significant strengths because the variables under study were specifically chosen for the prediction of morbidity and mortality, based on the TRPM. This contrasts with studies that utilize existing data retrospectively in relation to mortality where the scope of variables will be limited by the existing data, such as the recent study that utilized SCI Model Systems data [15]. The selection of variables also is more systematic using the conceptual model and, as a result, allows the findings to be interpreted from within the larger framework.

Second, we utilized health predictors based on previous research, so we measured health dimensions. This has both strengths and limitations. It is a strength that it is based on previous research and the variables, such as pressure ulcers, are not being measured by a single variable. This allows for a wider interpretation of the data that is not limited to a specific or specific indicators, but rather the underlying conceptual dimension (i.e., elevated risk of pressure ulcers). However, the translation is less direct. For instance, we did not independently identify the relationship of depression with mortality, yet depression was measured and incorporated under the general health dimension. Similarly, individual variables previously identified in relationship to allcause mortality, such as fractures and amputations [19], were not analyzed independent of the factors under which they were grouped. Clearly, this study only scratched the surface of the potential of linking health domains to mortality.

Third, use of a competing risk model with independent identification of risk and protective factors for each cause of mortality is a substantial strength. At the same time, this reduces the power of the analysis to identify significant differences. Therefore, we were restricted in the number of predictor variables that could be evaluated in a given analysis. We focused on health factors, which are one of the levels within the TRPM. Ultimately, each set of predictors within the conceptual model needs to be analyzed in relation to cause-specific mortality. This simply cannot be done without conducting a series of analyses and building the models, stage by stage.

Another decision related to the classification of causes of death. On an a priori basis, we chose the categories that have the greatest potential risk of excess mortality after SCI and, therefore, the greatest potential explanatory value. Other classifications, including larger categories, would have been possible. For instance, we focused on deaths due to unintentional injuries, rather than the broader category of external causes, so that we draw clear conclusions (all external causes would include unintentional injuries, suicide, and homicide). Similarly, we focused on pneumonia and influenza, rather than the broader category of respiratory causes, and septicemia rather than the broader category of infective and parasitic diseases. While this helped interpretation of our analyses, it did result in a large "other" 
category related to several risk and protective factors, which are difficult to interpret.

\section{Future research}

Additional research is clearly needed to better identify the risk and protective factors for the specific causes of mortality. This includes the analysis of other sets of predictors that may be of importance because they either predict types of mortality to identify individuals to serve as the targets for intervention strategies or because they identify specific targets for intervention. Whereas psychological factors, such as personality, might help identify individuals who should be targeted for prevention strategies, linking behaviors to cause-specific mortality will provide specific targets for behavioral change. Clearly, there is a need for augmenting sample sizes and study power, as well as investigation of a broader range of predictor variables, even within a given category, such as health parameters. It also will be important for further research to identify crosscutting themes that add conceptual clarity to the analysis and any discovery, with death due to despair being a primary example. It is only through continued research that we will be able to identify a wider array of precursors of excess mortality related to specific causes, so that we may target individuals at high risk and develop intervention strategies that may be implemented on an individualized basis.

\section{Conclusion}

Three health domains were significantly associated with mortality after SCI, and the patterns of association varied as a function of specific cause of death. The findings reinforce the importance of pressure ulcer and infection prevention, as well as facilitation of global health, including emotional well-being. As multiple disciplines may contribute to the overall health of people with SCI, multidisciplinary efforts are needed to promote longevity. Preventing morbidity appears to be the key to preventing each cause-specific type of mortality.

Funding The contents of the publication were developed under a grant from the National Institute on Disability, Independent Living, and Rehabilitation Research (NIDILRR grant number 90IF0066). NIDILRR is a Center within the Administration for Community Living (ACL), Department of Health and Human Services (HHS). The contents of this publication do not necessarily represent the policy of NIDILRR, ACL, HHS, and you should not assume endorsement by the Federal Government.

Author contributions YC was responsible for research method, results, and conclusion sections. ND was responsible for literature search and introduction. JK was responsible for discussion.

\section{Compliance with ethical standards}

Conflict of interest The authors declare that they have no conflict of interest.

Statement of ethics We certify that all applicable institutional and governmental regulations concerning the ethical use of human volunteers were followed during the course of this research.

Publisher's note: Springer Nature remains neutral with regard to jurisdictional claims in published maps and institutional affiliations.

\section{References}

1. DeVivo MJ, Krause JS, Lammertse DP. Recent trends in mortality and causes of death among persons with spinal cord injury. Arch Phys Med Rehabil. 1999;80:1411-9.

2. Strauss DJ, DeVivo MJ, Paculdo DR, Shavelle RM. Trends in life expectancy after spinal cord injury. Arch Phys Med Rehabil. 2006;87:1079-85.

3. NSCISC. Annual statistical report. Birmingham, AL: University of Alabama; 2017.

4. Chamberlain JD, Meier S, Mader L, von Groote PM, Brinkhof MW. Mortality and longevity after a spinal cord injury: systematic review and meta-analysis. Neuroepidemiology. 2015;44:182-98.

5. DeVivo MJ. Sir Ludwig Guttmann Lecture: trends in spinal cord injury rehabilitation outcomes from model systems in the United States: 1973-2006. Spinal Cord. 2007;45:713-21.

6. Shavelle RM, DeVivo MJ, Brooks JC, Strauss DJ, Paculdo DR. Improvements in long-term survival after spinal cord injury? Arch Phys Med Rehabil. 2015;96:645-51.

7. Osterthun R, Post MW, van Asbeck FW, van Leeuwen CM, van Koppenhagen CF. Causes of death following spinal cord injury during inpatient rehabilitation and the first five years after discharge. A Dutch cohort study. Spinal Cord. 2014;52:483-8.

8. Savic G, DeVivo MJ, Frankel HL, Jamous MA, Soni BM, Charlifue S. Causes of death after traumatic spinal cord injury-a 70-year British study. Spinal Cord. 2017;55:891-7.

9. Leite VF, Souza DR, Imamura M, Battistella LR. Post-discharge mortality in patients with traumatic spinal cord injury in a Brazilian hospital: a retrospective cohort. Spinal Cord. 2018;57: 134-40.

10. NSCISC. Spinal cord injury facts and figures at a glance. Birmingham, AL: University of Alabama; 2018.

11. Chamberlain JD, Gmunder HP, Hug K, Jordan X, Moser A, Schubert M, et al. Differential survival after traumatic spinal cord injury: evidence from a multi-center longitudinal cohort study in Switzerland. Spinal Cord. 2018;56:920-30.

12. McGrath R, Hall O, Peterson M, DeVivo M, Heinemann A, Kalpakjian C. The association between the etiology of a spinal cord injury and time to mortality in the United States: A 44-year investigation. J Spinal Cord Med. 2018:1-9. https://doi.org/10. 1080/10790268.2018.1505311. [Epub ahead of print]

13. Krause JS. Secondary conditions and spinal cord injury: A model for prediction and prevention. Top Spinal Cord Inj Rehabil. 1996;2:217-27.

14. Krause JS, Saunders LL, DiPiro ND, Reed KS. Theoretical risk and prevention model for secondary health conditions and mortality: 15 years of research. Top Spinal Cord Inj Rehabil. 2013;19:15-24.

15. Krause JS, Cao Y, DeVivo MJ, DiPiro ND. Risk and protective factors for cause-specific mortality after spinal cord injury. Arch Phys Med Rehabil. 2016;97:1669-78. 
16. Krause JS, Carter R, Zhai Y, Reed K. Psychologic factors and risk of mortality after spinal cord injury. Arch Phys Med Rehabil. 2009;90:628-33.

17. Krause JS, Carter RE. Risk of mortality after spinal cord injury: relationship with social support, education, and income. Spinal Cord. 2009;47:592-6.

18. Krause JS, Carter RE, Pickelsimer E. Behavioral risk factors of mortality after spinal cord injury. Arch Phys Med Rehabil. 2009;90:95-101.

19. Krause JS, Carter RE, Pickelsimer E, Wilson D. A prospective study of health and risk of mortality after spinal cord injury. Arch Phys Med Rehabil. 2008;89:1482-91.

20. Krause JS, DeVivo MJ, Jackson AB. Health status, community integration, and economic risk factors for mortality after spinal cord injury. Arch Phys Med Rehabil. 2004;85:1764-73.

21. Krause JS, Saunders LL. Risk of mortality and life expectancy after spinal cord injury: The role of health behaviors and participation. Top Spinal Cord Inj Rehabil. 2010;16:53-60.

22. Krause JS, Saunders LL. Health, secondary conditions, and life expectancy after spinal cord injury. Arch Phys Med Rehabil. 2011;92:1770-5.

23. Krause JS, Saunders LL. Do risk factors for mortality after spinal cord injury parallel those from the general USA population?, expanded abstract. Top Spinal Cord Inj Rehabil. 2012;18:113-7.

24. Krause JS, Saunders LL. Socioeconomic and behavioral risk factors for mortality: do risk factors observed after spinal cord injury parallel those from the general USA population? Spinal Cord. 2012;50:609-13.

25. Krause JS, Saunders LL, DeVivo MJ. Income and risk of mortality after spinal cord injury. Arch Phys Med Rehabil. 2011; 92:339-45.

26. Krause JS, Saunders LL, Zhai Y. Stability of predictors of mortality after spinal cord injury. Spinal Cord. 2012;50:281-4.
27. Krause JS, Zhai Y, Saunders LL, Carter RE. Risk of mortality after spinal cord injury: an 8-year prospective study. Arch Phys Med Rehabil. 2009;90:1708-15.

28. Krause JS, Cao Y, DiPiro ND, Cuddy E. Personality, high-risk behaviors, and elevated risk of unintentional deaths related to drug poisoning among individuals with spinal cord injury. Arch Phys Med Rehabil. 2018;99:1941-8.

29. Krause JS, Reed KS, McArdle JJ. A structural analysis of health outcomes after spinal cord injury. J Spinal Cord Med. 2010;33:22-32.

30. CDC. National Death Index 2017. Available from: https://www. cdc.gov/nchs/ndi/index.htm.

31. SAS Institute Inc. SAS for Windows. 9.4 ed. Cary, NC: SAS Institute; 2015.

32. Allison PD. Survival analysis using the SAS system: a practical guide. Cary, NC: SAS Institute; 2010.

33. Austin PC, Lee DS, Fine JP. Introduction to the analysis of survival data in the presence of competing risks. Circulation. 2016;133:601-9.

34. Li C, DiPiro ND, Krause J. A latent structural equation model of risk behaviors and pressure ulcer outcomes among people with spinal cord injury. Spinal Cord. 2017;55:553-8.

35. Flattau A, Blank AE. Risk factors for 90-day and 180-day mortality in hospitalised patients with pressure ulcers. Int Wound J. 2014;11:14-20.

36. Cox J. Pressure injury risk factors in adult critical care patients: A review of the literature. Ostomy Wound Manag. 2017;63:30-43.

37. Margolis DJ, Knauss J, Bilker W, Baumgarten M. Medical conditions as risk factors for pressure ulcers in an outpatient setting. Age Ageing. 2003;32:259-64.

38. Li C, DiPiro ND, Cao Y, Szlachcic Y, Krause J. The association between metabolic syndrome and pressure ulcers among individuals living with spinal cord injury. Spinal Cord. 2016;54:967-72. 